\title{
Hiperplasia endometrial
}

\author{
Bernardo Moreno Escallón*
}

\begin{abstract}
RESUMEN: El presente artículo es una revisión de la epidemiología, etiopatogenia, diagnóstico y tratamiento de la hiperplasia endometrial, en donde se pretende entregar al especialista una actualización de la clasificación, un esquema específico de manejo y seguimiento de esta condición, para qứe pueda hacer un enfoque más racional de la hiperplasia endometrial.
\end{abstract}

PALABRAS CLAVES: Hiperplasia, adenocarcinoma endometrial, receptores estrogénicos y progestacionales, atipia citológica, antígeno carcinoembrionario, acetato de medroxiprogesterona.

SUMMARY: The present article is a review of the epidemiology, etiology, physiopathology, diagnosis and treatment of the endometrial hyperplasia, which objective is to provide the specialist an up-to-date classification and specific work up and follow up of such condition, in order to carry out a rational treatment of the endometrial hyperplasia.

KEY WORDS: Hyperplasia, endometrial adenocarcinoma, estrogen and progesterone receptors, cytologic atypia, carcinoembryonic antigen, medroxyprogesterone acetate.

\section{Definición}

La hiperplasia endometrial es una condición histopatológica del endometrio a nivel de sus glándulas, epitelio, estroma y vasos sanguíneos, producida por un efecto persistente de los estrógenos no antagonizado en forma adecuada por la progesterona.

\section{Fisiopatología}

La hiperplasia endometrial está usualmente asociada con anovulación. El significado de este cambio en el endometrio está relacionado con la edad de la paciente. En la joven con hiperplasia endometrial quien ha menstruado cíclicamente, este cambio será más a menudo visto durante un período de anovulación frecuentemente asociado a stress. Igualmente en la mujer joven con algunos desórdenes endocrinos, como el que se aprecia en la enfermedad de ovario poliquístico, la hiperplasia endometrial refleja uną estimulación a largo plazo y persistente de estrógenos. La hiperplasia en la mujer premenopáusica no es raro observarla en asociación con falla ovárica, anovulación y producción persistente de estrógenos por el ovario. En la mujer postmenopáusica puede estar asociado con un aumento en la conversión endógena de androstenediona a estrona o excepcionalmente por la presencia de un tumor ovárico funcionante. La hiperplasia también puede ser inducida por el uso de estrógenos exógenos. La fuente exógena incluye el uso de estrógenos para tratar la menopausia o con menor frecuencia, otros estados hipoestrogénicos como la disgenesia gonadal (1-2). Según algunos investigadores, la hiperplasia aunque no ha sido un transtorno al que se haya sometido a investigación minuciosa, tiene su origen a la estimulación de estrógenos no antagonizada, o cuando menos antagonizada en forma insuficiente por la progesterona. En los casos en los que no hay trastorno en la relación estrógeno/progesterona y los ciclos son ovulatorios, la hiperplasia depende posiblemente de una anormalidad del "órgano terminal", como consecuencia de deficiencia de receptores que permitan sólo a los estrógenos actuar en las glándulas en forma anormal (3).

Durante muchos años la hiperplasia endometrial ha estado ligada con el cáncer de endometrio. Varios investigadores (46) han aportado evidencias bien documentadas sosteniendo que la hiperplasia glanduloquística, adenomatosa, atípica y el carcinoma in situ del endometrio, cuando no son tratados, pueden eventualmente evolucionar hacia un carcinoma invasor. La Figura 1 ilustra en forma esquemática la secuencia de los cambios progresivos en el endometrio que pueden llegar a ocasionar un adenocarcinoma invasor. El esquema se basa en el trabajo de Sommers y col. (7) y del cual se desprenden algunas conclusiones importantes. En primer lugar indica que las mujeres destinadas a presentar carcinoma endometrial, tienen fases progresivas de estimulación glandular que, hasta un punto dado, dependen de estrógenos. La eliminación del estrógeno ocasiona regresión glandular. El punto crítico de retorno es la fase conocida como hiperplasia adenomatosa atípica o carcinoma in situ. Según datos de estudios prospectivos, en promedio, $10 \%$ de las mujeres que tienen tal lesión, más tarde presentarán carcinoma invasor. Gusberg y col. (8) encontraron que el $18 \%$ de 562 mujeres con hiperplasia adenomatosa desarrollaron carcinoma de endometrio en un período de 1 a 30 años después de haberse hecho el diagnóstico inicial de la hiperplasia Wentz (9) reportó que un $14.6 \%$ de pacientes con hiperplasia quística, un $26 \%$ de las mujeres con hiperplasia adenomatosa, un $75 \%$ de las mujeres con hiperplasia atípica y un $100 \%$ de mujeres con carcinoma in situ de endometrio desarrollaron lesiones invasivas en un período de 1 a 4 años después de que la hiperplasia fue identificada. De acuerdo con Sherman y Brown (10) el seguimiento de 235 mujeres con diferentes lesiones precancerosas no tratadas, el $72 \%$ desarrolló carcinoma de endometrio en un período de 2 a 18 años. Parece ser que el 
PATOGENESIS DE LA HIPERPLASIA Y NEOPLASIA ENDOMETRIAL (Hiperestregenismo no antagonizado)
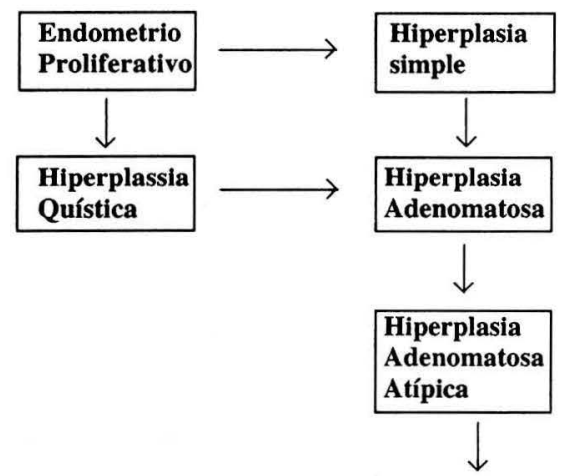

Adenocarcinoma
Figura 1

PREVENCION DE LA HIPERPLASIA Y NEOPLASIA ENDOMETRIAL

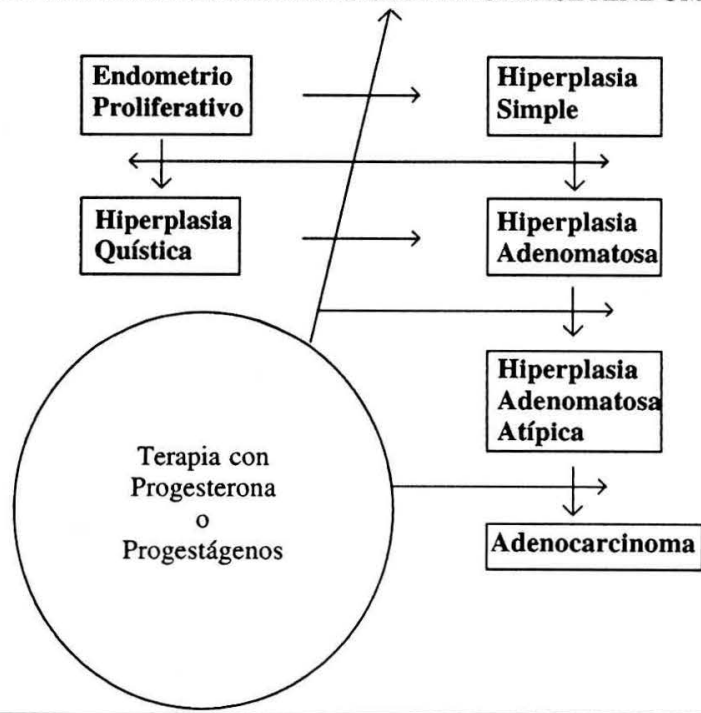

tiempo requerido para la transformación maligna de esas lesiones endometriales, es bastante variable.

Si se acepta que existe una relación entre la hiperplasia endometrial y el adenocarcinoma de endometrio, se puede aceptar que los factores de riesgo identificados para desarrollar esta última condición sean comunes también para la hiperplasia. Existe una serie de circunstancias en donde por aumento en exposición a los estrógenos endógenos, los pacientes se encuentran en mayor riesgo de desarrollar hiperplasia/Ca. Endometrial, como son (11-12):

\begin{tabular}{|l|c|c|}
\hline Diagnóstico & Prevalencia (\%) & RRA. \\
\hline $\begin{array}{l}\text { A. Tumores ováricos producto- } \\
\text { res de estrógenos (Prevalen- } \\
\text { cia: 3.5\% y Riesgo: 10) }\end{array}$ & 3,5 & 10 \\
\hline $\begin{array}{l}\text { B. Ovario androgénico } \\
\text { (Prevalencia: 19-25\%) RR= }\end{array}$ & $19-25$ & \\
\hline C. Obesidad (50-59 años) & & \\
\hline $\begin{array}{l}\text { 1. Exceso de peso de 20 a } \\
\text { 50 libras sobre el peso ideal: } \\
\text { Riesgo 3. }\end{array}$ & --- & 3 \\
\hline $\begin{array}{l}\text { 2. Exceso mayor de 50 libras } \\
\text { sobre el peso ideal: Riesgo 10 }\end{array}$ & $-\ldots-$ & 10 \\
\hline D. Nuliparidad: Riesgo 2-5 & --- & $2-5$ \\
\hline $\begin{array}{l}\text { E. Menopausia tardía: } \\
\text { Riesgo 2.4 }\end{array}$ & & \\
\hline F. Diabetes: Riesgo 2.8 & $-\ldots-$ & 2.8 \\
\hline $\begin{array}{l}\text { G. Enfermedades hepáticas } \\
\text { crónicas. RR }\end{array}$ & $-\ldots--$ & $-\ldots--$ \\
\hline
\end{tabular}

En la medida que la secreción de estrógenos ováricos disminuye en la menopausia y desâparece la incidencia de la acción antogénica de la progesterona (P4) de hiperplasia/Ca. endometrial aumenta. Sin embargo está bien establecido que la producción de estrógenos continúa en la mujer postmenopáusica. Vermeulen reporta que durante la menopausia todos los niveles de esteroides, excepto la estrona, son significativamente más bajos cuando son comparados con los niveles de la fase folicular de la pre-menopausia. El principal origen de la estrona es la conversión periférica de la androstenediona ovárica y adrenal. Un aumento en la capacidad de conversión periférica de la androstenediona a estrona ocurre en una variedad de situaciones, tales como la edad, la obesidad, enfermedad hepática crónica y disfunción tiroidea. Judd reportó como el aumento de la conversión periférica de androstenediona asociado a obesidad originaba un nivel elevado de estrógenos tanto en mujeres post-menopáusicas normales como en mujeres con carcinoma endometrial. Hausknecht y Gusberg, Riskallah y col., Calanog y col., también han reportado como la conversión periférica a estrona está aumentada en pacientes con cáncer endometrial. Sin embargo otros autores como MacDonald y col. no han encontrado ninguna diferencia en las tasas de conversión. Siiteri y MacDonald encontraron diferencias cuando incluyeron los pacientes de hiperplasia endometrial, en las series de los pacientes con cáncer endometrial (12).

Una producción aumentada de androstenediona puede también originar un aumento en la formación periférica de estrona. Las mujeres con enfermedad de ovario poliquístico producen tres o cuatro veces más androstenediona que las mujeres normales, y mayores cantidades periféricas de estrona. Los tumores funcionantes del ovario también están asociados a un aumento en la producción de androstenediona.

Existe alguna evidencia que los niveles de la globulina transportadora de hormonas sexuales (SHBG), se reduce en un 60 a $80 \%$ en mujeres obesas post-menopáusicas con y $\sin$ carcinoma endometrial y hay un aumento de 2 a 3 veces de los niveles de estradiol libre (Ref.). En mujeres obesas postmenopáusicas los niveles de estradiol libre pueden estar más disponibles al receptor citoplasmático en el órgano blanco, debido a la disminución de la SHBG. La información anterior provee una explicación de como los factores de riesgo, como la obesidad, nuliparidad, ovario poliquístico, tumores funcionantes del ovario, transtornos hepáticos y del tiroides, se relacionan con la hiperplasia/Ca. endometrial. Factores que aumentan o prolongan la exposición del endometrio a 
estrógenos producto de la conversión periférica de androstenediona, están asociados con un incremento en el riesgo de desarrollar hiperplasia/Ca. endometrial (12).

Los intentos fallidos para demostrar que existen niveles de esteroides sexuales aumentados en sangre periférica en pacientes con carcinoma endometrial cuando se comparan con pacientes normales, hace ver que una cosa son los niveles séricos de las hormonas esteroides y otra es la concentración de hormona libre y el efecto que produzcan a nivel del receptor citoplasmático y nuclear del tejido blanco esos mismos esteroides; como también de la capacidad individual de cada huésped de metabolizarlos y su susceptibilidad genética para desarrollar Ca. endometrial.

Por otra parte, mujeres que planifican con anticonceptivos orales secuenciales (hoy en día en desuso), post-menopáusicas así como mujeres jóvenes con disgenesia gonadal que necesitan terapia de reemplazo, sin la ingesta de progestágenos mayor de 10 a 14 días tienen un riesgo de 4 a 8 veces más de desarrollar hiperplasia/Ca. endometrial, que las mujeres que no están expuestas a terapia con estrógenos (13). Esta asociación está directamente relacionada con el tiempo de exposición y la dosis de estrógenos $(12,14-16)$.

Las células endometriales humanas contienen sistemas de receptores para estrógenos y progesterona. La forma como estos esteroides actúan es uniéndose a un receptor específico de tipo proteico localizado en el citoplasma. El complejo hormona-receptor se transloca al núcleo e interactúa con un aceptor poteico asociado con la cromatina. Hay un aumento en la síntesis del RNA y en la actividad de RNA polimerasa seguido por un aumento en la síntesis proteica (17). Esto finalmente resulta en un crecimiento del tejido y/o secreción de productos celulares. El principal efecto del estrógeno sobre el tejido endometrial es la proliferación celular y aumento en la concentración de receptores tanto para estrógenos (RE) como para progesterona (RP). En contraposición la progesterona reduce el nivel de receptores para estrógenos. Durante el ciclo menstrual el nivel máximo de RE ocurre en la fase previa a la ovulación y disminuyen en la fase secretora en tanto la mayor concentración de RP se encuentran durante la fase proliferativa tardía y la mínima concentración durante la fase secretora tardía. En otras palabras, los niveles de estos receptores se modifica de acuerdo con las concentraciones de los estrógenos y progesterona durante el ciclo menstrual normal. En la mujer post-menopáusica que recibe terapia estrogénica, se logran obtener niveles de RE similares a la fase proliferativa inicial, al cabo de 2 a 3 semanas de tratamiento. $\mathrm{Si}$ se agrega un progestágeno se disminuyen tanto los RE como los RP (18-19).

La hiperplasia endometrial y el carcinoma de endometrio contienen receptores para estradiol y progesterona. Los receptores en estos tejidos tienen esencialmente las mismas características físico-químicas de tamaño, punto isoeléctrico, especificidad esteroidea y afinidad que los RE y RP del endometrio normal. Se han encontrado niveles elevados de RP entre 60 a $90 \%$ de los especímenes con hiperplasia endometrial (20). La administración de progestágenos conduce a la reducción de la concentración de receptores citoplasmáticos tanto para estrógenos como para progesteronaen mujeres con hiperplasia endometrial. Esta es una de las bases del tratamiento que se describirá posteriormente.

\section{Clasificación}

La clasificación anatomopatológica de la hiperplasia endometrial se había venido haciendo con base en la propuesta por la OMS, la cual la clasificaba en: Hiperplasia quística, hiperplasia adenomatosa e hiperplasia atípica (21).

Hoy en día algunos patólogos la siguen utilizando y por eso es importante conocerla y ver la equivalencia con la clasificación actual, la cual se menciona más adelante. Kurman RJ y col. en 1985 hicieron un estudio retrospectivo de 170 pacientes con diferentes grados de hiperplasia endometrial, con el fin de evaluar las características histopatológicas de la hiperplasia y el comportamiento clínico-patológico de la misma, durante un período de 1 a 26 años. Las alteraciones citológicas y de la arquitectura de las glándulas, fueron analizadas en forma separada con el fin de evaluar su papel predictivo con relación a la probabilidad de progresión a carcinoma. En este estudio, la única carecterística histológica que demostró en forma significativa, ser de valor pronóstico, fue la presencia de atipia citológica, pues $2 \%$ de los pacientes con hiperplasia endometrial sin atipia, progresaron hacia carcinoma en un tiempo promedio de 9.5 años, mientras que un 23\% de los que tenían hiperplasia atípica progresaron hacia carcinoma en un tiempo promedio de 4.1 años (22). En otro estudio de tipo prospectivo, llevado a cabo por Ferency A. y Gelfand M. en el año 89, en 85 pacientes con hiperplasia que recibieron tratamiento médico con acetato de medroxiprogesterona, y que tuvieron un seguimiento entre 2 y 12 años, encontraron un $25 \%$ de progresión a carcinoma en las pacientes con hiperplasia atípica, mientras que no hubo progresión a carcinoma en ninguna de las pacientes con hiperplasia sin atipia (23). Así como éstos, existen otros estudios que demuestran que la presencia o ausencia de la atipia citológica en las hiperplasias es de valor pronóstico y por eso se debe tener en cuenta en el tratamiento del paciente.

Por las razones anteriores, ha surgido la nueva clasificación de la hiperplasia que es la siguiente:
A. Hiperplasia simple.
B. Hiperplasia compleja.
C. Hiperplasia simple atípica.
D. Hiperplasia compleja atípica.
Las cuales se definen a continuación:

\section{A. Hiperplasia simple}

Proliferación benigna caracterizada por aumento en el número de glándulas endometriales, las cuales pueden estar dilatadas, aglomeradas o conglutinadas en forma leve o moderada y no existe atipia citológica.

\section{B. Hiperplasia compleja}

Está caracterizada por las glándulas con contornos irregulares que demuestran una marcada estructura compleja, y que se aglomeran y disponen íntimamente "espalda contra espalda". Tampoco existe atipia citológica.

\section{Hiperplasia atípica}

Es una proliferación de glándulas que exhiben atipia citológica y en las cuales existen varios grados de atipia nuclear y pérdida de polaridad. La atipia citológica muestra agrandamiento nuclear, núcleo prominente y cromatina dispersa irregularmente. 
La atipia citológica en ausencia de hiperplasia compleja se denomina hiperplasia simple atípica, y la atipia citológica acompañada de un marcado número de glándulas aglomeradas y dispuestas íntimamente "espalda contra espalda", se denomina hiperplasia compleja atípica.

El carcinoma de endometrio bien diferenciado, se distingue de la hiperplasia atípica, por una invasión del estroma arbitrariamente definida. En forma breve, los cuatro criterios de invasión son los siguientes:

1. Infiltración irregular de las glándulas, asociado a un estroma fibroblástico alterado o con respuesta desmoplástica.

2. Un patrón glandular confluente en donde glándulas individuales interrumpidas por el estroma forman un patrón cribiforme.

3. Patrón papilar extenso.

4. Estroma reemplazado por masas de epitelio escamoso. Los criterios 2, 3 y 4 deben ser lo suficientemente extensos como para comprometer un $50 \%$ del campo $(2.1 \mathrm{~mm})$ de bajo poder mirado en un campo de $10 \mathrm{X}$ Pero la histología se estudia en $100 \mathrm{X}$.

La equivalencia de la clasificación actual, con la de la OMS (Organización Mundial de la Salud), es la siguiente:

\section{Clasificación hiperplasia endometrial}

\begin{tabular}{|ll|}
\hline \multicolumn{1}{|c|}{ OMS (Antigua) } & \multicolumn{1}{c|}{ Actual } \\
\hline Hiperplasia quística & Hiperplasia simple \\
Hiperplasia adenomatosa & Hiperplasia compleja \\
Hiperplasia atípica & Hiperplasia simple o \\
& compleja con atipia. \\
\hline
\end{tabular}

\section{Diagnóstico}

El diagnóstico de la hiperplasia endometrial es básicamente de tipo histológico y generalmente se llega a él a través de un legrado uterino o biopsia de endometrio practicada en aquellas pacientes con transtornos menstruales o hemorragias uterinas anormales; es un hallazgo en muchas ocasiones accidental.

En aquellos pacientes con riesgo elevado de desarrollar un carcinoma de endometrio, se han desarrollado una serie de técnicas y dispositivos para obtener especímenes endometriales; esto también podría aplicarse para el diagnóstico de la hiperplasia endometrial, por su conocida relación con el carcinoma.

El examen ideal para todo diagnóstico es áquel que tenga una buena sensibilidad y especificidad, poco costosa, fácil de realizar y que produzca el mínimo de molestia al paciente. Desafortunadamente no existe un examen que se pueda aplicar en forma rutinaria a grandes poblaciones y que sirva para el Dx de esta entidad. A continuación se mencionan una serie de técnicas utilizadas para diagnóstico de carcinoma de endometrio, con su respectivo porcentaje de confiabilidad y cuyos datos son extrapolables para el diagnóstico de la hiperplasia endometrial (11):

\begin{tabular}{|lc|}
\hline Técnica\% & Confiabilidad \\
\hline Curetaje por succión & 98.0 \\
Dilatación y curetaje & 96.2 \\
Biopsia de Endometrio & 90.0 \\
Aspiración Endometrial & 88.7 \\
Cepillado Endometrial & 87.4 \\
Lavado con presión negativa (Gralee-set) & 81.9 \\
\hline
\end{tabular}

Tradicionalmente la dilatación y curetaje fraccionado, ha sido el procedimiento para el diagnóstico definitivo del carcinoma endometrial/hiperplasia endometrial, especialmente en aquellos casos en donde la simple biopsia de endometrio ha sido insuficiente o cuando existe estenosis cervical, que requiera de dilatación bajo anestesia. Por otra parte sobre la base de la experiencia clínica disponible en el momento actual, la biopsia de endometrio parece ser la técnica más confiable y menos costosa para obtener una muestra para estudio histológico en primera instancia. En nuestra experiencia la correlación diagnóstica entre biopsia de endometrio y curetaje uterino es alrededor del $96 \%$ y por eso el método inicial de estudio para pacientes con hemorragia uterina o transtornos menstruales es la biopsia de endometrio (24).

Para llevar a cabo la biopsia de endometrio existen 3 tipos de curetas: Novak, Randall y Kevorkian. Para disminuir al máximo el dolor que produce la toma de la muestra, se recomienda hacer un bloqueo paracervical con 10 c.c. de xylocaina al $2 \%$ sin epinefrina colocadas a las 4 y 8 horas ( 5 cc en cada cuadrante). Si la paciente es colaboradora y tiene el cerviz dilatado o dilatable es posible que no se requiera de anestesia local.

Como las curetas mencionadas anteriormente son metálicas y por lo tanto rígidas no dejan de producir alguna molestia, por eso también se recomienda cuando sea posible, utilizar la cureta plástica de succión endometrial (desechable), que sólo tiene $3 \mathrm{~mm}$ de diámetro externo y con la cual se logra obtener una buena muestra con el mínimo de molestias para el paciente.

Hoy con el nuevo impulso que ha tomado la endoscopia pélvica, la histeroscopia ha vuelto a tomar importancia como instrumento diagnóstico en el estudio de sangrados uterinos anormales, ya que además de visualizar las lesiones orgánicas, se pueden tomar biopsias dirigidas de las mismas. A pesar de estas ventajas no deja de ser un procedimiento costoso, y que muchas veces requiere anestesia general para llevarla a cabo.

En resumen: para el diagnóstico de la hiperplasia endometrial, se llega a través de muestras endometriales obtenidas por dilatación y curetaje, biopsia de endometrio o biopsia dirigida con histeroscopia.

\section{Métodos de ayuda diagnóstica}

De la hiperplasia endometrial/Ca. de endometrio, en la actualidad se están realizando estudios con ultrasonografía, métodos de inmunohistoquímica, estudio de DNA y receptores entre otros, los cuales se mencionan más ampliamente a continuación:

\section{A. Ultrasonografía transvaginal}

Se utiliza para medir el espesor endometrial y de acuerdo con su correlación histológica, poder establecer en el futuro cuál podría ser el grosor que podría indicar patología endometrial. Existen algunos ejemplos al respecto: Nasri y col. encontraron en un estudio de 111 pacientes postmenopáusicas, en donde el $63 \%$ con endometrio atrófico, tenían un espesor endometrial de menos de $5 \mathrm{~mm}$ y el $31 \%$ con endometrio anormal, el grosor endometrial estaba por encima de $5 \mathrm{~mm}$ (25). Malpany A. y col. estudiaron 90 mujeres con hiperplasia endometrial y las compararon con 126 con 
endometrio normal, encontrando diferencias estadísticamente significantes en los espesores endometriales promedios, de 18.8 y $5.4 \mathrm{~mm}$ respectivamente (26). Ellos recomiendan como límite entre normalidad y anormalidad un espesor endometrial de $10 \mathrm{~mm}$. Por otra parte otros autores como Osner y Lin., recomiendan un límite de $8 \mathrm{~mm}$ y de ésta forma obtienen una especificidad del $100 \%$ y una sensibilidad del $81 \%(27-28)$.

Hoy por hoy para fines prácticos, cada institución debe establecer de acuerdo con su experiencia el valor de confiabilidad que le garantice una buena especificidad y sensibilidad con éste método.

\section{B. Inmunohistoquímica}

Los marcadores tumorales se están utilizando básicamente en el diagnóstico y seguimiento del adenocarcinoma de endometrio y eventualmente para monitorizar la progresión de lesiones premalignas como la hiperplasia hacia el carcinoma.

Se han hecho estudios comparativos entre la ferritina y el antígeno carcinoembrionario (ACE), encontrándose que el primero es mejor marcador biológico que el ACE en diferenciar entre endometrio hiperplásico y neoplásico (29).

En otro estudio de 153 pacientes, en el que se estudiaron especímenes normales, con hiperplasia atípica y carcinoma de endometrio, se compararon el CA 19-9, el CA 125 y el ACE, encontrándose un porcentaje de positividad para cáncer endometrial del $93 \%, 65 \%$ y $58 \%$ respectivamente; el porcentaje de positividad del Ca 19-9 para hiperplasia atípica fue bajo, mientras que el porcentaje de positividad fue paralelamente en aumento con el grado de diferenciación del tumor (30). Otroestudio de 47 pacientes con carcinoma de endometrio y 20 pacientes con hiperplasia endometrial llevado a cabo por Panici y col., encontraron un porcentaje de positividad de los marcadores séricos tumorales en cáncer de endometrio del $43 \%$ para CA $125,32 \%$ para CA $15-3$ y $14 \%$ para ACE. En los casos de hiperplasia endometrial el CA 15-3 y el ACE fueron normales y sólo hubo 1 caso positivo para CA 125 (31).

Estos y muchos otros ejemplos, muestran el valor relativo de los marcadores tumorales, y podrían eventualmente servir como coadyuvantes en el seguimiento y evolución de la hiperplasia endometrial.

\section{Tinción tisular}

La coloración de los mucopolisacáridos contenidos en especímenes endometriales se ha utilizado para diferenciar entre lesiones benignas, pre-malignas y malignas. Se han hecho estudios utilizando diferentes colorantes como el Azul alciano, el PAS y el Azul de toluidina, en endometrio secretor, hiperplasia quística, adenomatosa, atípica y adenocarcinoma, en forma comparativa. El citoplasma de las lesiones premalignas y malignas contienen mucopolisacáridos ácidos sulfatados y no sulfatados mientras que los tejidos con lesiones benignas contienen mucopolisacáridos neutros y glucógeno. Estas condiciones se han aprovechado, para demostrar como el citoplasma de los tejidos con hiperplasia atípica y adenocarcinoma de endometrio toman coloración con el Azul alciano a un $\mathrm{pH}$ de 2.5, mientras que no se observa dicha coloración cuando se trata de endometrio normal o con hiperplasia quística o adenomatosa sin atipias. Este colorante ha demostrado ser superior para hacer esas diferencias cuando ha sido comparado con el PAS y el Azul de toluidina (32).

\section{Receptores}

Los estudios de receptores estrogénicos y progestacionales, han demostrado patrones muy heterogéneos, tanto en hiperplasia endometrial como en carcinoma de endometrio. Esto ha hecho imposible establecer unos patrones uniformes que ayuden al manejo de esas condiciones.

Estudios en endometrio durante el ciclo menstrual han mostrado un contenido de receptores estrógenicos (RE) más elevado en el endometrio proliferativo; ese contenido disminuye gradualmente a través de la fase post-ovulatoria del ciclo menstrual tanto en el epitelio como en el estroma. Se han encontrado un contenido alto de receptores estrogénicos tanto en el epitelio como en el estroma de las hiperplasias, semejante al del endometrio proliferativo, mientras que el contenido de estos receptores ha sido bajo en el epitelio de los tejidos con hiperplasia atípica y carcinoma de endometrio. En esta última situación, se han encontrado RE bajos, en comparación con el endometrio proliferativo normal o hipleplásico, sin embargo, se han descrito casos en los cuales los RE del estroma son altos a pesar de carecer de RE en el epitelio (33).

\section{E. DNA ploide}

El estudio del ácido desoxirribonucleico (DNA) mediante la citometría de flujo en células procedentes de especímenes con patología benigna y maligna, está tratando de ser introducido en el manejo del cáncer en general, para poder ayudar a establecer un pronóstico y tratamiento adecuados. En el estudio de un tumor puede haber DNA procedente de una misma población celular y en este caso se habla de tumores diploides, mientras que si el tumor tiene más de una población celular identificable, se denomina aneuploide. En el estudio de citometría de flujo para el DNA, el grado de DNA aneuploide se calcula por índice de DNA, que es la proporción que existe entre el DNA de las células aneuploides del tumor y el contenido de DNA de las células diploides de la muestra (34).

La aneuploidía está relacionada con la pérdida de diferenciación del tumor. Entre más diferenciado el tumor es más baja la incidencia de aneuploidía. La presencia de aneuploidía en un carcinoma de endometrio predice una enfermedad más agresiva. Por otra parte la detección de aneuploidía en una hiperplasia endometrial atípica puede indicar una transformación a carcinoma endometrial (35).

\section{Tratamiento y manejo}

Volviendo a la definición de la hiperplasia endometrial mencionada en el primer capítulo, es lógico pensar que el manejo de la misma se haga con base en los progestágenos, los cuales se han venido utilizando desde la década de los años 50 , no solamente para el tratamiento de la hiperplasia endometrial sino también para el carcinoma de endometrio. En el año 51, Kelly (36) encontró que la terapia con progesterona era efectiva en el tratamiento del carcinoma de endometrio primario y metastásico. En 1959, Kistner (37) reportó que los progestágenos tenían un efecto benéfico sobre la hiperplasia del endometrio. Wentz y Eichner $(9,38)$ han confirmado la efectividad de varios tipos de progestágenos en 
el manejo de la hiperplasia endometrial. En 1953, Wellenback y Rakoff (39), demostraron en hamsters a los que se les había estirpado los ovarios, que la hiperplasia endometrial inducida, mostraba regresión rápida cuando se administraba progesterona. En 1956, aparecieron agentes progestacionales sintéticos que pudieron usarse en la investigación clínica. Las publicaciones de Kelley y Baker (40) despertaron interés al describir los efectos del Caproato de 17 alfa hidroxiprogesterona en la metástasis del cáncer endometrial. Gambrell y asociados reportaron que el tratamiento con progestágeno era efectivo en revertir el endometrio hiperplásico a normal en 242 de 258 pacientes $(92.8 \%)$ (12).

Entre los progestâgenos utilizados en el tratamiento de la hiperplasia endometrial se encuentran la dimetisterona, el megestrol y el acetato de medroxiprogesterona, los cuales han demostrado tener capacidad de hacer regresar las lesiones hiperplásicas del endometrio y lo hacen usualmente dentro de los tres meses de iniciada la terapia (41).

La administración de progesterona reduce el contenido de receptores para estrógenos en el útero de la rata (42). Otros autores han demostrado un efecto similar en el endometrio humano (43-44). Además de reducir el contenido de receptores estrogénicos, bloquean la nueva síntesis de estos receptores o reciclaje (45). Los progestágenos también incrementan la

Tabla 1

MANEJO ESQUEMATICO DE LA HIPERPLASIA ENDOMETRIAL

\begin{tabular}{|c|c|c|c|c|c|c|c|c|c|c|c|c|}
\hline \multirow{3}{*}{$\begin{array}{l}\text { EDAD } \\
\text { (años) }\end{array}$} & \multicolumn{6}{|c|}{ HIPERPLASIA SIN ATIPIA } & \multicolumn{6}{|c|}{ HIPERPLASIA CON ATIPIA } \\
\hline & \multicolumn{2}{|c|}{ D R M } & \multirow{2}{*}{\begin{tabular}{|c|} 
DA \\
$\mathbf{Y}$ \\
$\mathbf{U}$
\end{tabular}} & \multirow{2}{*}{ ND } & & & \multicolumn{2}{|c|}{ D R M } & \multirow{2}{*}{ DR } & \multirow{2}{*}{\begin{tabular}{|c|}
$\mathbf{D A}$ \\
$\mathbf{Y}$ \\
$\mathbf{U}$ \\
\end{tabular}} & \multirow{2}{*}{ ND } & \multirow{2}{*}{$\mathbf{R Q}$} \\
\hline & D F & NDF & & & & & DF & NDF & & & & \\
\hline$\ll 35$ & $\begin{array}{ll}\text { 1) } & (1) \\
\text { 2) } & \mathrm{EA}\end{array}$ & $\begin{array}{c}\text { 1) }(1) \\
\text { 2) } \\
\mathrm{AO}\end{array}$ & (1) & & & & $\begin{array}{ll}\text { 1) } & (2) \\
\text { 2) } & \mathrm{EA}\end{array}$ & $\begin{array}{c}\text { 1) }(2) \\
\text { 2) } \\
\mathrm{AO}\end{array}$ & & & & \\
\hline »35 & $\begin{array}{ll}\text { 1) } & (1) \\
\text { 2) } & \mathrm{EA}\end{array}$ & $\begin{array}{c}1(1) \\
2) \\
\mathrm{AH}\end{array}$ & (1) & (3) & $\mathbf{H A}$ & (3) & $\begin{array}{ll}\text { 1) } & (2) \\
\text { 2) } & \mathrm{AI}\end{array}$ & $\begin{array}{l}\text { 1) } \\
\text { 2) }\end{array}$ & $\begin{array}{l}\text { (2) } \\
\mathrm{AH}\end{array}$ & (3) & HA & (3) \\
\hline
\end{tabular}

CONVENCIONES

DF: Deseo de fertilidad

NDF: No deseo de fertilidad

DRM: Deseo de regularidad menstrual

DA y U: Deseo de amenorrea y conservación del útero

RQA: Riesgo quirúrgico alto

NDU: No deseo de conservación del útero

EA: Estudio de anovulación

AO: Anovulatorios orales

AH: Anticoncepción hormonal

HA: Histerectomía abdominal

AI: Análisis individual en cada caso

(1) Esquema 1 de tratamiento

(2) Esquema 2 de tratamiento

(3) Esquema 3 de tratamiento (Ver texto) actividad de la enzima 17 Beta-estradiol dehydrogenasa la cual metaboliza preferencialmente el estradiol a estrona, la cual tiene mucho menos potencia biológica y por lo tanto menos efecto estimulante sobre el endometrio (46). También restauran la integridad de la membrana basal del endometrio hiperplásico y disminuyen el factor de crecimiento epidérmico a nivel del receptor tanto en el tejido normal como en el hiperplásico (47).

El progestágeno más recomendado hoy en día para el tratamiento de las hiperplasias, es el acetato de medroxiprogesterona (Provera (R), el cual es un derivado sintético de la progesterona y por lo tanto posee los mecanismos de acción mencionados anteriormente. En una experiencia propia iniciada en el año 1983 y publicada en 1991 (24), se utilizó el acetato de medroxiprogesterona de depósito el cual mostraba algunas ventajas sobre los otros progestágenos tales como: Acción prolongada con una dosis total a un plazo relativamente corto; inhibición de las gonadotrofinas, reduciendo así la producción de andrógenos precursores en el ovario: inhibición leve de la producción de andrógenos suprarrenales; alteración mínima o ninguna sobre las lipoproteínas de alta densidad (HDL), y aumento de la tasa de depuración de la testosterona, con disminución de su producción en suero (53-54). En ese estudio se encontró una excelente respuesta histológica, con reversión de la hiperplașia en el $100 \%$ de los casos un mes después de iniciada la terapia (24).

El manejo específico de la hiperplasia endometrial depende de los siguientes criterios:

A. Edad

B. Tipo de hiperplasia

C. Deseode regular el ciclo menstrual o tratar la amenorrea

D. Deseo de fertilidad

E. Deseo de conservar el útero y evitar la cirugía

F. Riesgo quirúrgico del paciente.

Los esquemas de tratamiento médico de la hiperplasia endometrial se pueden resumir en:

A. Esquema 1: Acetato de medroxiprogesterona $10 \mathrm{mgr} /$ día 12 días, durante 3 a 6 meses. Luego biopsia de endometrio un mes después de finalizado el tratamiento.

B. Esquema 2: Acetato de medroxiprogesterona $20 \mathrm{mgr} /$ día en forma continua durante 6 meses. Luego biopsia de endometrio un mes después de finalizado el tratamiento. Si hay mejoría, se continúa con el esquema 1.

C. Esquema 3: Acetato de medroxiprogesterona 150 mgs I.M. dos veces por semana durante 8 semanas. Luego biopsia de endometrio a los 3 y 6 meses de finalizado el tratamiento. El seguimiento se hará con biopsia cada año.

En la Tabla 1 se muestra en forma esquemática el tratamiento inicial de la hiperplasia endometrial de acuerdo con los criterios especificados anteriormente y en algunas situaciones especiales.

\section{Seguimiento}

Si después del esquema de tratamiento 1 no existe regresión de las lesiones histológicas, o existe progresión de las mismas, se pasará al esquema 2 de tratamiento. Se practicará biopsia de endometrio un mes después de finalizado el tratamiento. Si hay mejoría o regresión histológica de la lesión y la paciente es menor de 35 años y desea fertilidad, 
se le practicará estudio etiológico de la anovulación y luego se hará el tratamiento específico de la misma o se indicarán inductores de ovulación de acuerdo con los esquemas ya fijados previamente (48). Si la paciente es menor de 35 años, no tiene deseo de fertilidad y presenta una hiperplasia sin atipia, si la evolución después del tratamiento es satisfactoria, continuará con anovulatarios orales combinados oinyectables de depósito. ¿Por quéno se continúa indefinidamente con el esquema 1 que ustedes proponen? además de los anteriores. Si ésta misma paciente presenta hiperplasia atípica y no existe respuesta satisfactoria con el esquema 2 , se pasará al esquema 3 , luego se seguirá con controles de biopsia endometrial a los 3, 6 y 12 meses de terminado el tratamiento. El seguimiento se continuará luego de control anual de biopsia.

La paciente con hiperplasia atípica que responde satisfactoriamente con el esquema 2 y no desee fertilidad, se le recomendará después de finalizado el tratamiento que continúe con el esquema 1 y protección con métodos de barrera, o con anovulatorios orales combinados, si la evolución después de ésta segunda fase de tratamiento es satisfactoria, se hará biopsia de control como se mencionó anteriormente.

En las pacientes mayores de 35 años que han sido sometidas al esquema 3 de tratamiento y no haya habido ni respuesta clínica ni histológica adecuada se aumentará la dosis de medroxiprogesterona de depósito a 300 mgrs. 2 veces por semana por 8 semanas más. Si a pesar de ésto no hay mejoría, la paciente será candidata para histerectomía abdominal total.

Es importante dejar en claro en éste momento, que la hiperplasia endometrial como tal con o sin atipias, no es indicación para llevar a cabo Ooforectomía bilateral, ya que al extraer solamente el útero, queda resuelto el problema del riesgo de desarrollar un adenocarcinoma de endometrio. (En la Tabla 1 se muestra en forma esquemática el tratamiento inicial de la hiperplasia endometrial de acuerdo con los criterios especificados anteriormente, y en algunas situaciones específicas).

Las pacientes en esquema 3 de tratamiento que presentan mejoría histológica, con cierta frecuencia consultan por manchado genital intermitente o continuo. En estos casos se recomienda dar un manejo inicial con acetato de noretisterona (Primolut-nor) a dosis de 10 mgrs 2 veces al día durante 15 días y luego continuar con manejo cíclico a dosis de 10 mgrs. diarios por 12 días mensualmente por 3 a 6 meses, en caso necesario.

Otra situación que se puede presentar durante el manejo de la hiperplasia es que haya mejoría histológica, pero la paciente continúa con hemorragias abundantes. En este caso es posible que haya necesidad de practicar dilatación y curetaje uterinos e histeroscopia diagnóstica que pueda descartar o confirmar una posible causa orgánica del sangrado.

En términos generales, se observa que durante el tratamiento y seguimiento de la hiperplasia endometrial, cuando no existe una mejoría clínica, es muy posible que la paciente tenga otra causa orgánica adicional causante de sus hemorragias uterinas y por eso es importante investigar la causa para poder llevar a cabo el tratamiento específico.

Como recomendaciones especiales para el manejo inicial de la paciente, es importante explicarle y discutir con ella, el tipo de tratamiento que se va a llevar a cabo, para que en caso que no se produzca el resultado esperado, la paciente esté dispuesta a continuar el paso siguiente con un manejo un poco más agresivo. Ṕor eso es importante enseñarle ciertas cifras estadísticas que la ayudarán a escoger el mejor tratamiento. El paciente debe saber que la evolución de la hiperplasia sin atipia es diferente a la hiperplasia atípica, y que cuando se encuentre en la primera situación, las posibilidades de regresión histológica con el tratamiento médico serán alrededor del $80 \%$ con un $20 \%$ de persistencia y las probabilidades de progresión a carcinoma de endometrio serán de un $2 \%$ en un promedio de 9 años (22-23). En contraste, cuando exista hiperplasia atípica el porcentaje de regresión con tratamiento médico será entre el 25 y $50 \%$, el de persistencia de 30 a $75 \%$ y las probabilidades de progresión a carcinoma endometrial de un $25 \%$ en un promedio de tiempo de 4 años (22-23). Otra alternativa que queda por plantear con base en los estudios de Kurman (22), es que un $30 \%$ de los pacientes a quienes se les hace el diagnóstico de hiperplasia atípica o sin atipia mediante la dilatación y curetaje iniciales quedan aparentemente tratadas con este procedimiento y no requieren tratamiento hormonal adicional. Esta última alternativa quedaría a criterio del médico tratante porque es muy difícil predecir prospectivamente cual va a ser la evolución histológica de determinada paciente.

Finalmente vale la pena mencionar algunos otros tratamientos que se han utilizado en el manejo de la hiperplasia, como el danazol a dosis de $400 \mathrm{mgr} / \mathrm{dí} / 3$ meses (49), el uso de la T-nova a base de levonorgestrel promete ser un DIU muy útil en el manejo de esta condición (50).

En los casos donde la histerectomía para el tratamiento de la hiperplasia o del sangrado uterino médicamente intratable sea considerada de riesgo quirúrgico alto, existe la alternativa de la ablación del endometrio mediante el resectoscopio (51) o el uso del láser de Nd: YAG (Neomidum) (52).

Finalmente, se están haciendo estudios sobre el valor de la prueba de desafío de progesterona (53) comparándola con la biopsia de endometrio para poder establecer en un futuro si el método hormonal no invasivo sería útil en el seguimiento a largo plazo de las pacientes tratadas para hiperplasia endometrial.

\section{BIBLIOGRAFIA}

1. Kistner RW., Hammond CH.B., Kaufman RH. Menopause Course. Sylabus of the American Fertility Society. XI Annual postgraduate course, Houston Texas, 1980; 19: 7.

2. Schiff I., Sela HK., Cramer D., Tulchinsky D., Ryan K. Endometrial hyperplasia in women on cyclic or continuous estrogen regimens. Fertil Steril 1982; 37: 79.
3. Scully RE. Definición de las alteraciones precursoras del carcinoma endometrial. Clínicas Obstétricas y Ginecológicas de Norteamérica. Editorial Interamericana 1982; 41-63.

4. Gusberg SB. Precursors corpus carcinoma: Estrogens and adenomatous hyperplasia. Am. J. Obstet. Gynecol. 1947; 54: 905. 
5. Hertig AT., Sommers SC. Genesis of endometrial cancer. I study of prior biopsies. Cancer 1949; 2: 946.

6. Vellios F., Endometrial hyperplasia and carcinoma in situ. Gynecol. Oncol. 1974; 2: 152.

7. Sommers SC., Hertig AT., Bengaloff H. Genesis of endometrial carcinoma: 11 cases $19-35$ years old. Cancer 1949; 2: 957.

8. Gusberg SB., Chen SY. and Cohen GJ. Endometrial Cancer: Factors influencing the chaise of treatment. Gynecol. Oncol. 1974; 2: 308.

9. Wentz WB. Treatment of persistent endometrial hyperplasia with progestins. Am. J. Obstet. Gynecol. 1966; 96: 999.

10. Sherman AI. and Brown S. The precursors of endometrial carcinoma. Am. J. Obstet. Gynecol. 1979; 135: 947.

11. Lifshitz S., Buchsbaum HJ. Diagnosis and staging of endometrial carcinoma. Posgraduate Obstetrics and Gynecology. 1983; 3: 1 .

12. Gambrell RD., Bagnell CA., Greenblatt RB. Role of estrogens and progesterone in the etiology and prevention of endometrial cancer: Review. Am. J. Obstet. Gynecol. 1983; 146: 696.

13. CramerDW., Knapp RC. Review of epidemiologic studies of endometrial cancer and exogenous estrogens. Obstet. Gynecol. 1979; 54: 521.

14. Henderson BE. The cancer question: an overview of recent epidemiologic and retrospective data. Am. J. Obstet. Gynecol. 1989; 161: 1859.

15. Bhagavan B., Parmley TH., Rosenshen NB., Jefferys J., Grisso J., Stolley P. Comparison of estrogen-induced hyperplasia to endometrial carcinoma Obstet. Gynecol. 1984; 64: 12.

16. Hammond CB., Maxon WS. Current status of estrogen therapy for the menopause. Fertil Steril 1982; 37: 5.

17. O'Malley B.W. Mechanisms of action of steroid hormones. N. Engl. J. Med. 1971; 284: 370 .

18. King RJB., Whiteheal MI., Campbell S. Effect of estrogen and progestin treatments on endometrium from postmenopausal women. Cancer Res 1979; 39: 1094

19. King RJB., Dyer G., Collins WP. Intracellular estradiol, estrone and estrogen receptor levels in endometria from posmenopausal women receiving estrogens and progestins. J. Steroid Biochem 1980; 13: 337.

20. Mac Laughlin DT. and Richarson GS. Progesterone binding by normal and abnormal human endometrium. J. Clin. Endocrinol. Metab. 1976; 52: 667.

21. Tavassoli F., Kraus FT. Endometrial lesions in uteri resected for atypical endometrial hyperplasia. Am. J. Clin. Pathol. 1978; 70: 770.

22. Kurman RJ., Kaminski PF., Norris HJ. The behavior of endometrial hyperplasia. Cancer 1985; 56: 403.

23. Ferenczy A., Gelfand M. The biologic significance of cytologic atypia in progestogen-treated endometrial hyperplasia. Am. J. Obstet. Gynecol. 1989; 160: 126.

24. Moreno-Escallón B., Angulo A., Botero LF., Restrepo P. Manejo de la hiperplasia endometrial con acetato de medroxiprogesterona de depósito. Rev. Col. Obstet. Ginecol. 1991; 42: 63.

25. Nasri MN., Shepard JH., Setchell ME., Lowe DG., Chard T. The role of vaginal scan in measurement of endometrial thickness in postmenopausal women. Br. J. Obstet. Gynecol. 1991; 98: 470.

26. Malpani A., Singer J., Wolverson MK., Merenda G. Endometrial hyperplasia: value of endometrial thickness in ultrasonographic diagnosis and clinical significance. J. Clin. Ultrasound. 1990; 18: 173.

27. Osmer R., Volksen M., Schaner A. Vaginosography for early detection of endometrial carcinoma. Lancet. 1990; 335: 1569.

28. Lin CM., Gonsink BB., Wolf IS., Feldesman RM., Stuenkel AC., Braly SP., Pretorius HD.: Endometrial thickess after menopause: Effect of hormone replacement. Radiology 1991; 180: 427.

29. Tsionou C., Minaretzis D., Papageorgiou I., Nakopoulou L., Michalas S., Aravantinos D. Expression of carcino embryonic antigen and feritin in normal, hyperplastic and neoplastic endometrium. Gynecol. Oncol. 1991; 41: 193.

30. Neunteufel W., Breitenecker G. CA 19-9, CA 125 and CEA in the endometrial mucosa during the mestrual cycle in atypical hyperplasia and endometrial carcinoma. Cancer-Lett 1989; 48: 77.
31. Panici PB., Scambia G., Baiocchi G., Perrone L., Greggi S., Battaglia F., Mancuso S. Multiple serum markers in patients with endometrial cancer. Gynecol. Obstet. Invest. 1989; 27: 208.

32. Laurie M., Elmalach Y., Weill S. The pattern of epithelial mucin secretion in normal hyperplastic and adenocarcinomatous endometrium. Gynecol. Oncol. 1988; 30: 274.

33. Bergeron C., Ferency A., Shyamala G. Distribution of estrogen receptors in various cell types of normal hiperplastic and neoplastic human endometrial tissues. Lab. Invest. 1988; 58: 338.

34. Iversen $\mathrm{OE}$. Flow cytometric deoxy ribonucleic acid index: A prognostic factor in endometrial carcinoma. Am. J. Obstet. Gynecol. 1986; 155: 770 .

35. Kysela B., Siracky J., Redecha M., Bardos A. Flow cytometry analysis of endometrial hyperplasia and carcinoma. Neoplasma. 1990; 37: 489.

36. Kelly RM. In proceeding of the Second Conference of Steroids and Cancer, council of pharmacy and chemistry, American Medical Association, Chicago 1951; 116.

37. Kistner RW. Histological effects of progestins on hyperplasia and carcinoma in situ of the endometrium. Cancer 1959; 12: 1106.

38. Eichner E., Abellera M. Endometrial hyperplasia treated by progestins. Obstet. Gynecol. 1971; 38: 739 .

39. Wellenback DL., Rakoff AE. Hyperplasia of the endometrium. J. Albert Einsten Med. Center. 1953; 2: 3.

40. Kelley RW., Baker WH. Effects of 17 alpha hydroxyprogesterone caproate on metastasic endometrial cancer. Conference on experimental clinical cancer chemotherapy, monograph 9, Bethesda, National Cancer Institute, 1965; 235.

41. Gal D., Edman CD., Vellios F., Forney J. Long-term effect of megestrol acetate in the treatment of endometrial hyperplasia. Am. J. Obstet. Gynecol. 1983; 146: 316.

42. Hsueh AJW., Peck EJ., Clark JH. Progesterone antagonism of the estrogen receptor and estrogen induced uterine growth. Nature 1975; 254: 337.

43. Tseng L., Gurpide E. Effect of progestins on estradiol receptor levels in human endometrium. J. Clin. Endocrinol Metab. 1975; 41: 402.

44. Kokko E., Janne O., Kauppila A., Vihko R. Effects of tamoxifen, medroxiprogesterone acetate and their combination on human endometrial estrogen and progestin receptor concentrations, 17 betahydroxysteroid dehydrogenase activity and serum hormone concentrations. Am. J. Obstet. Gynecol. 1982; 143: 382.

45. Clark JH., Anderson JN., Peck EJ. Estrogen receptor anti-estrogen complex: Atypical binding by uterine nuclei and effects on uterine growth. Steroids. 1974; 22: 707

46. Friedrich ER., Meyer JS. Estrogen-progestin pharmacodynamics of the postmenopausal endometrium studied by thymidine labeling. Am. J. Obstet. Gynecol. 1982; 143: 352.

47. Jasonni VM., Bulletti C., Balducci, Naldi S., Martinelli G., Galassi A., Flamigni $\mathrm{C}$. The effect of progestin on factors influencing growth and invasion of the endometrial carcinoma. Ann. N.Y. Acad. Sci. 1991; 622: 463.

48. Moreno-Escallón B. Inductores de ovulación. Universitas Médica 1989; 30: 6 .

49. Bulletti C., Jasonni U.M., Tabanelli S., Balduca M., Fuschini G., Flamigni C. Danazol reverses endometrial hyperplasia to normal endometrium. Acta Eur Fertil 1987; 18: 185.

50. Scarselli G., Tantini C., Colafranceschi M., Taddei GL., Bargelli G., Venturini N., Branconi F. Levonorgestrel nova $T$ and precancerous lesions of the endometrium. Eur J. Gynecol. Oncol. 1988; 9: 284.

51. De Cherney A., Diamond MP., Lavy G. Endometrial ablation for intractable uterine bleeding: Hysteroscopic resection. Obstet. Gynecol. 1987; 70: 668 .

52. Loffer FD. Hysteroscopic endometrial ablation with the Nd: YAG laser using a non-touch technique. Obstet. Gynecol. 1987; 69: 679.

53. Hanna JH., Major MC., Brady KW., Hill JM., Phillips LG. Detection of postmenopausal women at risk for endometrial carcinoma by a progesterone challange test. Am. J. Obstet. Gynecol. 1983; 147: 872. 